P. Orlik and L. Solomon

Nagoya Math. J.

Vol. 109 (1988), 23-45

\title{
DISCRIMINANTS IN THE INVARIANT THEORY OF REFLECTION GROUPS
}

\author{
PETER ORLIK AND LOUIS SOLOMON*
}

\section{§1. Introduction}

Let $V$ be a complex vector space of dimension $l$ and let $G \subset G L(V)$ be a finite reflection group. Let $S$ be the $C$-algebra of polynomial functions on $V$ with its usual $G$-module structure $(g f)(v)=f\left(g^{-1} v\right)$. Let $R$ be the subalgebra of $G$-invariant polynomials. By Chevalley's theorem there exists a set $\mathscr{B}=\left\{f_{1}, \cdots, f_{l}\right\}$ of homogeneous polynomials such that $R=$ $C\left[f_{1}, \cdots, f_{l}\right]$. We call $\mathscr{B}$ a set of basic invariants or a basic set for $G$. The degrees $d_{i}=\operatorname{deg} f_{i}$ are uniquely determined by $G$. We agree to number them so that $d_{1} \leq \cdots \leq d_{l}$. The map $\tau: V / G \rightarrow C^{l}$ defined by

$$
\tau(G v)=\left(f_{1}(v), \cdots, f_{l}(v)\right)
$$

is a bijection. Each reflection in $G$ fixes some hyperplane in $V$. Let $\mathscr{A}=\mathscr{A}(G)$ be the set of reflecting hyperplanes and let

$$
\begin{gathered}
N(G)=\bigcup_{H \in \infty} H \\
M(G)=V-\bigcup_{H \in \infty} H .
\end{gathered}
$$

If $H \in \mathscr{A}$ let $e_{H}$ be the order of the (cyclic) subgroup fixing $H$ and let $\alpha_{H} \in V^{*}$ be a linear form with kernel $H$. Since $\prod_{H \in \mathcal{A}} \alpha_{H}^{e H} \in R$ we may define a polynomial $\Delta\left(T_{1}, \ldots, T_{l} ; \mathscr{B}\right)$ in the indeterminates $T_{1}, \cdots, T_{l}$ by

$$
\Delta\left(f_{1}, \cdots, f_{l} ; \mathscr{B}\right)=\prod_{H \in \mathscr{\infty}} \alpha_{H H}^{e_{H}} .
$$

We call the polynomial $\Delta\left(T_{1}, \cdots, T_{l} ; \mathscr{B}\right)$ the discriminant of $G$ relative to $\mathscr{B}$ since it depends on the basic invariants. The hypersurface

$$
\tau(N(G) / G)=\left\{\left(z_{1}, \cdots, z_{l}\right) \in C^{l} \mid \Delta\left(z_{1}, \cdots, z_{l} ; \mathscr{B}\right)=0\right\}
$$

Received February 6, 1986.

* This work was supported in part by the National Science Foundation. 
will be called the discriminant locus. E. Bannai [1] computed the fundamental group of the complement $\tau(M(G) / G)$ of the discriminant locus for all irreducible unitary reflection groups with $\operatorname{dim} V=2$. In the course of this work she computed the discriminants $\Delta\left(f_{1}, f_{2} ; \mathscr{B}\right)$.

If $V_{R} \subset V$ is a real form of $V$ and $W \subset G L\left(V_{R}\right)$ is a finite Coxeter group we may view $W \subset G L(V)$ as a unitary reflection group. In an earlier paper [14] we defined a class of finite irreducible unitary reflection groups, called Shephard groups. In (2.11) we associate to each Shephard group $G \subset G L(V)$ a finite irreducible Coxeter group $W \subset G L(V)$ which is determined up to isomorphism. Both $G$ and $W$ are isomorphic to quotients of the same Artin group. Since $W$ is also a reflection group it has basic invariants, discriminant, etc. When both groups $G, W$ are in question we use notation such as $f_{i}^{G}, f_{i}^{W}, \Delta_{G}, \Delta_{W}, d_{i}^{G}, d_{i}^{W}$, etc. to indicate the dependence on $G, W$. If a statement involves only one Shephard group we usually suppress this dependence and may apply the statement to $G$ or $W$. Given a Shephard group $G$ and the corresponding Coxeter group $W$, inspection of the known values of $d_{i}^{G}, d_{i}^{W}$ as listed in Table 1 reveals the remarkable fact that

$$
d_{1}^{G} / d_{1}^{W}=\cdots=d_{l}^{G} / d_{l}^{W} .
$$

This suggests that there may be connections between the invariant theory of $G$ and $W$. Corollary (2.26) asserts that with suitable basic sets $\mathscr{B}_{G}$, $\mathscr{B}_{W}$ for $G, W$ the discriminant loci $\tau(N(G) / G)$ and $\tau(N(W) / W)$ are defined by the same polynomial:

$$
\Delta_{G}\left(T_{1}, \cdots, T_{l} ; \mathscr{B}_{G}\right) \approx \Delta_{W}\left(T_{1}, \cdots, T_{l} ; \mathscr{B}_{W}\right) .
$$

Here, and in the rest of this paper it is convenient to write $a \approx b$ if $b \in C^{*} a$. In (5.1) we use (1.7) and work of Deligne [5] to show that

(1.8) If $G \subset G L(V)$ is a Shephard group then $M(G)$ is a $K(\pi, 1)$ space.

We illustrate (1.6) and (1.7) for the pair $(G, W)$ where $G=G_{25}$ in the Shephard-Todd classification [19] and $W$ is the Coxeter group of type $A_{3}=D_{3}$. The degrees $d_{i}^{G}$ are $6,9,12$ and the degrees $d_{i}^{W}$ are $2,3,4$. Thus $d_{i}^{G} / d_{i}^{W}=3$ does not depend on $i$. To illustrate (1.7) we use polynomials $C_{6}, C_{9}, C_{12}$ and $\mathfrak{S}_{12}$ defined by Maschke [9, p. 326]. Shephard and Todd [19, p. 286] remarked that we may choose $\mathscr{B}_{G}=\left\{C_{6}, C_{9}, C_{12}\right\}$ and that $\mathfrak{\subseteq}_{12} \approx \prod_{H \in \mathscr{S}(G)} \alpha_{H}$. It follows from Maschke's work $\left[9\right.$, p. 326] that $\mathfrak{\Im}_{12}^{3}=$ 
Table 1.

\begin{tabular}{|c|c|c|c|c|}
\hline & \multicolumn{2}{|c|}{$G$} & \multicolumn{2}{|c|}{$W$} \\
\hline$S \& T$ & $\Gamma(G)$ & $d_{i}^{G}$ & $\Gamma(W)$ & $d_{\imath}^{W V}$ \\
\hline$G(p, 1, l)$ & $\stackrel{p}{0}{ }^{4}-0-\cdots-0$ & $p, 2 p, \cdots, l p$ & $0 \stackrel{4}{-} 0-\cdots-0$ & $2,4, \cdots, 2 l$ \\
\hline 3 & $\begin{array}{l}p \\
0\end{array}$ & $p$ & 0 & 2 \\
\hline 4 & $\begin{array}{lr}3 & 3 \\
0 & -0\end{array}$ & 4,6 & $0 \longrightarrow 0$ & 2,3 \\
\hline 8 & $\begin{array}{lr}4 & 4 \\
0 & 4\end{array}$ & 8,12 & $0-0$ & 2,3 \\
\hline 16 & $\begin{array}{lr}5 & 5 \\
0 & -0\end{array}$ & 20,30 & $0-0$ & 2,3 \\
\hline 5 & $\begin{array}{lll}3 & 4 & 3 \\
0 & 4\end{array}$ & 6,12 & $0 \stackrel{4}{-} 0$ & 2,4 \\
\hline 10 & $\begin{array}{lll}4 & & 3 \\
0 & - & 3 \\
- & 0\end{array}$ & 12,24 & $0 \stackrel{4}{-} 0$ & 2,4 \\
\hline 18 & $\begin{array}{lll}5 & 4 & 3 \\
0 & 3 \\
& 0\end{array}$ & 30,60 & $0 \stackrel{4}{-} 0$ & 2,4 \\
\hline 20 & $\begin{array}{lll}3 & & 3 \\
0 & -5 & 0\end{array}$ & 12,30 & $0{ }^{5}-0$ & 2,5 \\
\hline 6 & $\stackrel{3}{0} \stackrel{6}{-}_{0}$ & 4,12 & $0 \stackrel{6}{-} 0$ & 2,6 \\
\hline 9 & $\stackrel{4}{0} \stackrel{6}{-}_{0}$ & 8,24 & $0 \stackrel{6}{-} 0$ & 2,6 \\
\hline 17 & $\begin{array}{ll}5 & 6 \\
0 & \end{array}$ & 20,60 & $0 \stackrel{6}{6}^{-} 0$ & 2,6 \\
\hline 14 & $\begin{array}{l}3 \\
0\end{array}$ & 6,24 & $0 \stackrel{8}{0} 0$ & 2,8 \\
\hline 21 & $\stackrel{3}{0} \stackrel{10}{-} 0$ & 12,60 & $0 \stackrel{10}{-} 0$ & 2,10 \\
\hline 25 & $\begin{array}{rrr}3 & 3 & 3 \\
0 & -0\end{array}$ & $6,9,12$ & $0-0-0$ & $2,3,4$ \\
\hline 26 & 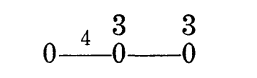 & $6,12,18$ & $0 \stackrel{4}{-} 0-0$ & $2,4,6$ \\
\hline 32 & $\begin{array}{rrrr}3 & 3 & 3 & 3 \\
0 & 0 & 0\end{array}$ & $12,18,24,30$ & $0-0-0-0$ & $2,3,4,5$ \\
\hline
\end{tabular}

$\left(432 C_{9}^{2}-C_{6}^{3}+3 C_{6} C_{12}\right)^{2}-4 C_{12}^{3}$ and thus

$$
\Delta_{G}\left(T_{1}, T_{2}, T_{3} ; \mathscr{B}_{G}\right)=\left(432 T_{2}^{2}-T_{1}^{3}+3 T_{1} T_{3}\right)^{2}-4 T_{3}^{3} .
$$

To compute the discriminant for $W=D_{3}$ choose a basis $x_{1}, x_{2}, x_{3}$ for $V^{*}$ such that $\prod_{H \in \mathscr{A}(W)} \alpha_{H}=\left(x_{1}^{2}-x_{2}^{2}\right)\left(x_{1}^{2}-x_{3}^{2}\right)\left(x_{2}^{2}-x_{3}^{2}\right)$. In this coordinate system $p_{1}=x_{1}^{2}+x_{2}^{2}+x_{3}^{2}, p_{2}=x_{1} x_{2} x_{3}$ and $p_{3}=x_{1}^{2} x_{2}^{2}+x_{1}^{2} x_{3}^{2}+x_{2}^{2} x_{3}^{2}$ is a basic set for $W$. Consider a cubic polynomial with roots $x_{1}^{2}, x_{2}^{2}, x_{3}^{2}$. The formula for the discriminant of this cubic, expressed in terms of the elementary symmetric functions of the roots, gives the identity 


$$
\prod_{H \in \mathscr{S}(W)} \alpha_{H I}^{e_{H}}=\left(2 p_{1}^{3}-9 p_{1} p_{3}+27 p_{2}^{2}\right)^{2}-4\left(p_{1}^{2}-3 p_{3}\right)^{3} .
$$

Let $f_{1}=p_{1}, f_{2}=p_{2} / 4$ and $f_{3}=p_{1}^{2}-3 p_{3}$. Then $\mathscr{B}_{W}=\left\{f_{1}, f_{2}, f_{3}\right\}$ is a basic set for $W$ and we have

$$
\Delta_{W}\left(T_{1}, T_{2}, T_{3} ; \mathscr{B}_{W}\right)=\left(432 T_{2}^{2}-T_{1}^{3}+3 T_{1} T_{3}\right)^{2}-4 T_{3}^{3} .
$$

Comparison of (1.9) and (1.11) illustrates (1.7) for this pair of groups $(G, W)$.

In (2.18) we define, for each Shephard group $G$ and basic set $\mathscr{B}$, an $l \times l$ matrix $\Delta\left(T_{1}, \cdots, T_{l} ; \mathscr{B}\right)$ with entries in $C\left[T_{1}, \cdots, T_{l}\right]$. We call $\Delta\left(T_{1}, \cdots, T_{l} ; \mathscr{B}\right)$ the discriminant matrix of $G$ with respect to $\mathscr{B}$. It follows from the definition of the discriminant matrix that

$$
\Delta\left(T_{1}, \cdots, T_{l} ; \mathscr{B}\right) \approx \operatorname{det} \Delta\left(T_{1}, \cdots, T_{l} ; \mathscr{B}\right) .
$$

Suppose $\overline{\mathscr{B}}=\left\{\bar{f}_{1}, \cdots, \bar{f}_{l}\right)$ is another basic set. Define polynomials $\varphi_{j}\left(T_{1}\right.$, $\left.\cdots, T_{l}\right)$ by $\bar{f}_{j}=\varphi_{j}\left(f_{1}, \cdots, f_{l}\right)$. Let $\bar{T}_{j}=\varphi_{j}\left(T_{1}, \cdots, T_{l}\right)$. It follows from (1.4) that

$$
\Delta\left(\bar{T}_{1}, \cdots, \bar{T}_{l} ; \overline{\mathscr{B}}\right) \approx \Delta\left(T_{1}, \cdots, T_{l} ; \mathscr{B}\right) .
$$

The corresponding transformation formula for discriminant matrices is more complicated; see (2.22). Write $\kappa=d_{\imath}^{G} / d_{\imath}^{W}$ for the constant defined in (1.6). The main result (2.25) of this paper implies that if $G$ is a Shephard group and $W$ is the associated Coxeter group then there exist basic sets $\mathscr{B}_{G}$ and $\mathscr{B}_{W}$ such that

$$
\Delta_{G}\left(T_{1}, \cdots, T_{l} ; \mathscr{B}_{G}\right)=\Delta_{W}\left(\kappa T_{1}, \cdots, \kappa T_{l} ; \mathscr{B}_{W}\right) .
$$

Since (1.14) asserts an equality of matrices, it is a much sharper statement than (1.7), which asserts an equality of polynomials. We derive (1.7) from (1.14) in (2.26). Our proof of (1.14) uses the classification of Shephard groups. For the exceptional groups with $\operatorname{dim} V=3,4$ we used MACSYMA on a VAX computer to find the discriminant matrix. We comment on the methods in Section 4 and give the results in the Appendix.

The discriminant $\Delta\left(T_{1}, \cdots, T_{l} ; \mathscr{B}\right)$ is a weighted homogeneous polynomial. We show in (5.13) that if $G$ is any irreducible unitary reflection group then the weights of $A\left(T_{1}, \ldots, T_{l} ; \mathscr{B}\right)$ are unique. We use this to show that (1.7) implies (1.6).

We would like to thank Ben Noble for introducing us to MACSYMA, and the Mathematics Research Center of the University of Wisconsin 
for providing computer time. We also want to thank Hiroaki Terao for many helpful conversations.

\section{§ 2. The Comparision Theorem}

In this section we state the main result of this paper, Theorem (2.25). In the first part of this section we assume that $G \subset G L(V)$ is any finite unitary reflection group and use the notation and the results of our earlier paper [14]. Let $\operatorname{Der}_{S}$ be the $S$-module of derivations of $S$ and let $\Omega_{S}=\operatorname{Hom}_{S}\left(\operatorname{Der}_{S}, S\right)$ be the $S$-module of differential 1-forms. Let $\left\{e_{i}\right\}$ be a basis for $V$ and let $\left\{x_{i}\right\}$ be the dual basis for $V^{*}$, fixed throughout this paper. Then $\left\{D_{i}=\partial / \partial x_{i}\right\}$ and $\left\{d x_{i}\right\}$ are bases for $\operatorname{Der}_{S}$ and $\Omega_{S}$ as $S$-modules. These modules are graded as follows. Give $S=\oplus_{p \geq 0} S_{p}$ its usual grading so that $S_{1}=V^{*}$. We call nonzero elements of $S$ forms of degree $p$. Grade $\operatorname{Der}_{S}$ by $\theta \in\left(\operatorname{Der}_{S}\right)_{q}$ if $\theta S_{p} \subseteq S_{p+q}$ for all $p$ and grade $\Omega_{S}$ by $\omega \in\left(\Omega_{S}\right)_{q}$ if $\omega\left(\left(\operatorname{Der}_{S}\right)_{p}\right) \subseteq S_{p+q}$ for all $p$. Thus $D_{i}$ has degree -1 and $d x_{i}$ has degree +1 . Both $\operatorname{Der}_{S}$ and $\Omega_{S}$ are $G$-modules. The $G$-action is given by $(g \theta)(f)=g\left(\theta\left(g^{-1} f\right)\right)$ and $(g \omega)(\theta)=g\left(\omega\left(g^{-1} \theta\right)\right)$ where $\theta \in \operatorname{Der}_{S}$ and $\omega \in \Omega_{S}$.

Recall from [14] that the $R$-module $\Omega_{S}^{G}$ of $G$-invariant 1 -forms is free of rank $l$ with a basis of homogeneous elements $d f_{1}, \cdots, d f_{l}$. The $R$ module $\operatorname{Der}_{S}^{G}$ of $G$-invariant derivations is free of rank $l$ with a basis $\mathscr{D}=\left\{\theta_{1}, \cdots, \theta_{l}\right\}$ of homogeneous elements. We call $\mathscr{D}$ a set of basic derivations. Note that $\operatorname{deg}\left(d f_{i}\right)=d_{i}=m_{i}+1$ where the $m_{i}$ are the exponents of $G$. Similarly $\operatorname{deg} \theta_{i}=n_{i}-1$ where the $n_{i}$ are the coexponents of $G$ as in $[12,14]$. We agree to number the $n_{i}$ in increasing order so that we have

$$
m_{1} \leq \cdots \leq m_{l} \text { and } n_{1} \leq \cdots \leq n_{l} .
$$

Define the Jacobian matrix $\mathbf{J}=\mathbf{J}\left(f_{1}, \cdots, f_{l}\right)$ by $\mathbf{J}_{i j}=D_{i} f_{j}$. If $\theta \in \operatorname{Der}_{s}$ then $\theta=\sum\left(\theta x_{i}\right) D_{i}$. Define a matrix $\mathbf{Q}=\mathbf{Q}\left(\theta_{1}, \cdots, \theta_{i}\right)$ by $\mathbf{Q}_{i j}=\theta_{j} x_{i}$. It follows from the definition of the $G$-action that if $f \in R$ and $\theta \in \operatorname{Der}_{S}^{G}$ then $\theta(f) \in R$. Since

$$
\left(\mathbf{J}^{T} \mathbf{Q}\right)_{i j}=\sum\left(\theta_{j} x_{k}\right)\left(D_{k} f_{i}\right)=\theta_{j} f_{i}
$$

it follows that $\mathbf{J}^{T} \mathbf{Q} \in M_{l}(R)$ where $\mathbf{J}^{T}$ is the transpose of $\mathbf{J}$. Let $T_{1}, \cdots, T_{l}$ be indeterminates. Since $R=C\left[f_{1}, \cdots, f_{l}\right]$ and the basic invariants $f_{1}, \cdots, f_{l}$ are algebraically independent, there exist unique polynomials $\psi_{i j}\left(T_{1}, \cdots\right.$, $\left.T_{l}\right) \in C\left[T_{1}, \cdots, T_{l}\right]$ such that $\left(\mathbf{J}^{T} \mathbf{Q}\right)_{i j}=\psi_{i j}\left(f_{1}, \cdots, f_{l}\right)$. 
(2.3) Definition. Let $\mathscr{B}=\left\{f_{1}, \cdots, f_{l}\right\}$ be a set of basic invariants and let $\mathscr{D}=\left\{\theta_{1}, \cdots, \theta_{l}\right\}$ be a set of basic derivations. Define the discriminant matrix $\Delta\left(T_{1}, \cdots, T_{l} ; \mathscr{B}, \mathscr{D}\right)$ by $\Delta\left(T_{1}, \cdots, T_{l} ; \mathscr{B}, \mathscr{D}\right)_{i j}=\psi_{i j}\left(T_{1}, \cdots, T_{l}\right)$. Thus

$$
\Delta\left(f_{1}, \cdots, f_{l} ; \mathscr{B}, \mathscr{D}\right)=\mathbf{J}\left(f_{1}, \cdots, f_{l}\right)^{T} \mathbf{Q}\left(\theta_{1}, \cdots, \theta_{l}\right) .
$$

Define homogeneous polynomials $J$ and $Q$ by

$$
J=\prod_{H \in \mathscr{\infty}} \alpha_{H}^{e_{H}-1} . \quad Q=\prod_{H \in \mathscr{Q}} \alpha_{H} .
$$

The degree of $J$ is the number $m$ of reflections in $G$. The degree of $Q$ is the number $n$ of reflecting hyperplanes [12, 14]. It is proved in [19] that $J \approx \operatorname{det} \mathbf{J}\left(f_{1}, \cdots, f_{l}\right)$ and in $[14,2.28]$ that $Q \approx \operatorname{det} \mathbf{Q}\left(\theta_{1}, \cdots, \theta_{l}\right)$. It follows from (1.4), (2.4) and (2.5) that

$$
\Delta\left(f_{1}, \cdots, f_{l} ; \mathscr{B}\right) \approx \operatorname{det} \Delta\left(f_{1}, \cdots, f_{l} ; \mathscr{B}, \mathscr{D}\right) \approx J Q .
$$

Thus $\Delta\left(T_{1}, \cdots, T_{l} ; \mathscr{B}, \mathscr{D}\right)$ depends on $\mathscr{B}$ and $\mathscr{D}$ but its determinant depends only on $\mathscr{B}$. For Shephard groups there is a natural choice of $\mathscr{D}$ in terms of $\mathscr{B}$. We repeat their definition [14]. Shephard [17,18] introduced the notion of a regular complex polytope $\mathscr{P}$ and showed that its symmetry group $G=\operatorname{Aut}(\mathscr{P})$ is an irreducible unitary reflection group. A regular convex polytope in $R^{l}$ defines a regular complex polytope in $V=C^{l}$ by scalar extension. If $\mathscr{P}$ arises in this way we say that $\mathscr{P}$ has a real form. In this case $G$ is a finite irreducible Coxeter group. Not all finite irreducible Coxeter groups arise in this way.

(2.7) Definition. A Shephard group is the symmetry group of a regular complex polytope.

Coxeter [4, pps. 94-5, 147-9] showed that for each Shephard group $G$ there exist generating reflections $s_{1}, \cdots, s_{l}$ and integers $p_{1}, \cdots, p_{l}$ and integers $q_{1}, \cdots, q_{l-1}$ such that $G$ has a presentation with defining relations:

$$
\begin{gathered}
s_{j}^{p_{j}}=1 \\
s_{j} s_{k}=s_{k} s_{j} \quad \text { if }|j-k| \geq 2 \\
s_{j+1} s_{j} s_{j+1} \cdots=s_{j} s_{j+1} s_{j} \cdots \quad \text { if } 1 \leq j \leq l-1
\end{gathered}
$$

where there are $q_{j}$ terms on each side of (2.10). Coxeter associated the symbol $p_{1}\left[q_{1}\right] p_{2} \cdots p_{l-1}\left[q_{l-1}\right] p_{l}$ to the group $G$. It follows from the clas- 
sification of Shephard groups that the symbol is uniquely determined by $G$ up to replacement of $p_{l}\left[q_{1}\right] p_{2} \cdots p_{l-1}\left[q_{l-1}\right] p_{l}$ by its reversal $p_{l}\left[q_{l-1}\right] p_{l-1}$ $\cdots p_{2}\left[q_{1}\right] p_{1}$. Thus we may make the following definition.

(2.11) Definition. If $G \subset G L(V)$ is a Shephard group with symbol $p_{1}\left[q_{1}\right] p_{2} \cdots p_{l-1}\left[q_{l-1}\right] p_{l}$, let $W \subset G L(V)$ be the Coxeter group with symbol $2\left[q_{1}\right] 2 \cdots 2\left[q_{l-1}\right] 2$. We call $W$ the Coxeter group associated to $G$.

The group $W$ is uniquely determined by $G$ up to conjugacy in $G L(V)$. If $\mathscr{P}$ has a real form then $W=G$.

(2.12) Definition. Let $G \subset G L(V)$ be a Shephard group with symbol $p_{1}\left[q_{1}\right] p_{2} \cdots p_{l-1}\left[q_{l-1}\right] p_{l}$. Associate to $G$ a graph $\Gamma(G)$ which has vertices $s_{1}, \cdots, s_{l}$ with labels $p_{1}, \cdots, p_{l}$ and edges between vertices $s_{j}$ and $s_{j+1}$ which are labeled $q_{j}$.

These graphs were introduced by Coxeter. If $W$ is a Coxeter group, let $\Gamma(W)$ be its Coxeter graph. In Table 1 we list the pairs $(G, W)$ in (2.11) together with their graphs. We omit the labels $p_{j}=2$ and $q_{j}=3$. Table 1 also contains the invariant degrees $d_{i}^{G}, d_{i}^{W}$. In [2] Coxeter noted in case $l=2$ and $q=q_{1}$ that

$$
d_{1}^{G}=2 h / q \quad \text { and } \quad d_{2}^{G}=h
$$

where $h$ is the order of $s_{1} s_{2}$. Since $d_{1}^{W}=2$ and $d_{2}^{W}=q$ this implies (1.6) in case $l=2$. In fact if $G$ is any Shephard group and $h^{G}$ is the order of $s_{1} s_{2} \cdots s_{l}$ then a case by case check shows that $d_{i}^{G} / d_{i}^{W}=h^{G} / h^{W}$ where $h^{W}$ is the Coxeter number of $W$. Since the order of the center $Z(G)$ is the greatest common divisor of $d_{1}^{G}, \cdots, d_{l}^{G}$ [20, Cor. 3.3] we get a stronger form of (1.6):

$$
\kappa=d_{i}^{G} / d_{i}^{W}=|Z(G)||| Z(W) \mid=h^{G} / h^{W} .
$$

Now we return to the invariant theory and the discriminant for a Shephard group. Let $G \subset G L(V)$ be a Shephard group and let $f_{1}$ be an invariant form of minimal positive degree. The main result of [14] is that the $R$-linear map $\operatorname{Hess}\left(f_{1}\right): \operatorname{Der}_{S}^{G} \rightarrow \Omega_{S}^{G}$ defined by

$$
\operatorname{Hess}\left(f_{1}\right): \theta \rightarrow \sum \theta\left(D_{i} f_{1}\right) d x_{i} \quad \theta \in \operatorname{Der}_{S}^{G}
$$

is an isomorphism of graded $R$-modules. In particular it follows that for given basic invariants $f_{1}, \cdots, f_{l}$ there exist unique basic derivations $\theta_{1}, \cdots, \theta_{l}$ with 


$$
\operatorname{Hess}\left(f_{1}\right) \theta_{i}=m_{1} d f_{i} \quad i=1, \cdots, l .
$$

The matrix of Hess $\left(f_{1}\right)$ relative to the pair of bases $\left\{D_{i}\right\}$ and $\left\{d x_{i}\right\}$ is the Hessian matrix $\mathbf{H}\left(f_{1}\right)$ defined by $\mathbf{H}\left(f_{1}\right)_{i j}=D_{i} D_{j} f_{1}$. The formula (2.16) is equivalent to the matrix equation

$$
\mathbf{H}\left(f_{1}\right) \mathbf{Q}\left(\theta_{1}, \cdots, \theta_{l}\right)=m_{1} \mathbf{J}\left(f_{1}, \cdots, f_{l}\right) .
$$

(2.18) Definition. Let $G$ be a Shephard group and let $\mathscr{B}=\left\{f_{1}, \cdots, f_{l}\right\}$ be a set of basic invariants. If $\mathscr{D}=\left\{\theta_{1}, \cdots, \theta_{l}\right\}$ satisfies (2.17) we call $\mathscr{D}$ the set of basic derivations associated to $\mathscr{B}$ and define

$$
\Delta\left(T_{1}, \cdots, T_{l} ; \mathscr{B}\right)=\Delta\left(T_{1}, \cdots, T_{l} ; \mathscr{B}, \mathscr{D}\right) .
$$

We call $\Delta\left(T_{1}, \cdots, T_{l} ; \mathscr{B}\right)$ the discriminant matrix of $G$ with respect to $\mathscr{B}$.

(2.19) Lemma. (i) $\boldsymbol{\Delta}\left(f_{1}, \cdots, f_{l} ; \mathscr{B}\right)=m_{1} \mathbf{J}^{T} \mathbf{H}\left(f_{1}\right)^{-1} \mathbf{J}$;

(ii) $\Delta\left(T_{1}, \cdots, T_{l} ; \mathscr{B}\right)$ is a symmetric matrix;

(iii) $\theta_{i} f_{\jmath}=\theta_{j} f_{i}$ for $1 \leq i, j \leq l$.

Proof. Formula (i) follows from (2.4) and (2.17); (ii) follows from (i); (iii) follows from (i) and (2.2).

We showed in $[14,2.23]$ that $\theta_{1}=\sum x_{i} D_{i}$ is the Euler derivation. It follows from (2.19. iii) that $\theta_{1} f_{j}=\theta_{i} f_{1}=d_{j} f_{j}$. It follows from (2.19. ii) that

$$
\Delta\left(T_{1}, \cdots, T_{l} ; \mathscr{B}\right)=\left(\begin{array}{cccc}
d_{1} T_{1} & d_{2} T_{2} & \cdots & d_{l} T_{l} \\
d_{2} T_{2} & * & \cdots & * \\
\vdots & \vdots & & \vdots \\
d_{l} T_{l} & * & & *
\end{array}\right)
$$

For convenience we define a matrix $\mathbf{D}\left(T_{1}, \cdots, T_{l} ; \mathscr{B}\right)$ by

$$
\mathbf{D}\left(T_{1}, \cdots, T_{l} ; \mathscr{B}\right)=\Delta\left(T_{1} / d_{1}, \cdots, T_{l} / d_{l} ; \mathscr{B}\right) .
$$

We also call $\mathbf{D}\left(T_{1}, \cdots, T_{l} ; \mathscr{B}\right)$ the discriminant matrix of $G$ with respect to $\mathscr{B}$. Note that

$$
\mathbf{D}\left(d_{1} f_{1}, \cdots, d_{l} f_{l} ; \mathscr{B}\right)=\Delta\left(f_{1}, \cdots, f_{l} ; \mathscr{B}\right) .
$$

In the next lemma we compare $\mathbf{D}\left(T_{1}, \cdots, T_{l} ; \mathscr{B}\right)$ and $\mathbf{D}\left(T_{1}, \ldots, T_{l}, \overline{\mathscr{B}}\right)$ for basic sets $\mathscr{B}, \mathscr{B}$. In the argument we use the fact proved in $[14,5.4]$ that if $G$ is a Shephard group, then an invariant form of minimal positive degree is unique up to a constant multiple. 
(2.22) Lemma. Let $\mathscr{B}=\left\{f_{1}, \cdots, f_{l}\right\}$ and $\overline{\mathscr{B}}=\left\{\bar{f}_{1}, \cdots, \bar{f}_{l}\right\}$ be basic sets for the Shephard group $G$. Define $c \in C^{*}$ by $\bar{f}_{1}=c f_{1}$. Let $\mathbf{E}$ be the $l \times l$ diagonal matrix $\mathbf{E}=\operatorname{diag}\left(d_{1}, \cdots, d_{l}\right)$. Let $p_{3}$ be the polynomial defined by $d_{j} \bar{f}_{j}=p_{j}\left(d_{1} f_{1}, \cdots, d_{l} f_{l}\right)$. Let $\bar{T}_{\jmath}=p_{3}\left(T_{1}, \cdots, T_{l}\right)$. Let $\mathbf{M}$ be the $l \times l$ matrix with $(k, j)$ entry $\mathbf{M}_{k j}=\partial \bar{T}_{j} / \partial T_{k}$. Let $\mathbf{N}=\mathbf{E M E}^{-1}$. Then

$$
\mathbf{D}\left(\bar{T}_{1}, \cdots, \bar{T}_{l} ; \overline{\mathscr{B}}\right)=c^{-1} \mathbf{N}^{T} \mathbf{D}\left(T_{1}, \cdots, T_{l} ; \mathscr{B}\right) \mathbf{N} .
$$

Proof. Write $\mathbf{J}(\mathscr{B})=\mathbf{J}\left(f_{1}, \cdots, f_{l}\right)$. A calculation using the chain rule gives $\mathbf{J}(\overline{\mathscr{B}})=\mathbf{J}(\mathscr{B}) \mathbf{N}$. Thus we get $\mathbf{D}\left(d_{1} \bar{f}_{1}, \cdots, d_{l} \bar{f}_{l} ; \overline{\mathscr{B}}\right)=m_{1} \mathbf{J}(\overline{\mathscr{B}})^{T} \mathbf{H}\left(\bar{f}_{1}\right)^{-1} \mathbf{J}(\overline{\mathscr{B}})$ $=m_{1} \mathbf{N}^{T} \mathbf{J}(\mathscr{R})^{T} \mathbf{H}\left(\bar{f}_{1}\right)^{-1} \mathbf{J}(\mathscr{B}) \mathbf{N}$. Since $\bar{f}_{1}=c f_{1} \quad$ we have $\mathbf{H}\left(\bar{f}_{1}\right)=c \mathbf{H}\left(f_{1}\right)$ so $\mathbf{D}\left(d_{1} \bar{f}_{1}, \cdots, d_{l} \bar{f}_{l} ; \overline{\mathscr{B}}\right)=c^{-1} \mathbf{N}^{T} \mathbf{D}\left(d_{1} f_{1}, \cdots, d_{l} f_{l} ; \mathscr{B}\right) \mathbf{N}$.

Now we are in position to compare the discriminant matrices $\mathbf{D}_{G}\left(T_{1}\right.$, $\left.\cdots, T_{l} ; \mathscr{B}\right)$ of a Shephard group $G$ and $\mathbf{D}_{W}\left(T_{1}, \cdots, T_{l} ; \mathscr{B}_{W}\right)$ of the associated Coxeter group $W$.

(2.24) Notation. Let $G \subset G L(V)$ be a Shephard group and let $W$ be the corresponding Coxeter group. Given basic sets $\mathscr{B}_{G}$ and $\mathscr{B}_{W}$ we sometimes write $\mathscr{B}_{G} \sim \mathscr{B}_{W}$ if $\mathbf{D}_{G}\left(T_{1}, \ldots, T_{l} ; \mathscr{B}_{G}\right)=\mathbf{D}_{W}\left(T_{1}, \ldots, T_{l} ; \mathscr{B}_{W}\right)$.

The main result of this paper, proved in Sections 3 and 4 , is the following comparison theorem for discriminant matrices of $G$ and $W$. In view of (2.20) this implies (1.14).

(2.25) THEOREM. Let $G \subset G L(V)$ be a Shephard group and let $W$ be the corresponding Coxeter group. (i) If $\mathscr{B}_{W}$ is a basic set for $W$ then there exists a basic set $\mathscr{B}_{G}$ for $G$ such that $\mathscr{B}_{G} \sim \mathscr{B}_{W}$. (ii) If $\mathscr{B}_{G}$ is a basic set for $G$ then there exists a basic set $\mathscr{B}_{W}$ for $W$ such that $\mathscr{B}_{G} \sim \mathscr{B}_{W}$.

(2.26) Corollary. Let $G \subset G L(V)$ be a Shephard group and let $W$ be the corresponding Coxeter group. Then there exist basic sets $\mathscr{B}_{G}, \mathscr{B}_{W}$ such that

$$
\Delta_{G}\left(T_{1}, \cdots, T_{l} ; \mathscr{B}_{G}\right) \approx \Delta_{W}\left(T_{1}, \cdots, T_{l} ; \mathscr{B}_{W}\right) .
$$

Proof. By (2.25) there exist basic sets $\overline{\mathscr{B}}_{G}=\left\{f_{1}^{G}, \cdots, f_{l}^{G}\right\}$ and $\overline{\mathscr{B}}_{W}=$ $\left\{f_{1}^{W}, \cdots, f_{l}^{W}\right\}$ such that $\overline{\mathscr{B}}_{G} \sim \overline{\mathscr{B}}_{W}$. Thus $\mathbf{D}_{G}\left(T_{1}, \cdots, T_{l} ; \overline{\mathscr{B}}_{G}\right)=\mathbf{D}_{W}\left(T_{1}, \cdots, T_{l}\right.$, $\left.\overline{\mathscr{B}}_{W}\right)$. It follows from $(2.20)$ that

$$
\Delta_{G}\left(T_{1} / d_{1}^{G}, \ldots, T_{l} / d_{l}^{G} ; \overline{\mathscr{B}}_{G}\right)=\Delta_{W}\left(T_{1} / d_{1}^{W}, \ldots, T_{l} / d_{l}^{W} ; \overline{\mathscr{B}}_{W}\right) .
$$

Let $\mathscr{B}_{G}=\left\{d_{1}^{G} f_{1}^{G}, \cdots, d_{l}^{G} f_{l}^{G}\right\}$ and let $\mathscr{B}_{W}=\left\{d_{1}^{W} f_{1}^{W}, \cdots, d_{l}^{W} f_{l}^{W}\right\}$. The assertion follows from (1.13) by taking determinants. 
The next proposition shows that it suffices to prove (2.25) for one particular basic set $\mathscr{B}_{\mathrm{W}}$ in (i) and one particular basic set $\mathscr{B}_{G}$ in (ii).

(2.27) Proposition. Suppose there exist basic sets $\mathscr{B}_{W}, \mathscr{B}_{G}$ for $W, G$ such that $\mathscr{B}_{G} \sim \mathscr{B}_{W}$. (i) If $\overline{\mathscr{B}}_{W}$ is any basic set for $W$ then there exists a basic set $\overline{\mathscr{B}}_{G}$ for $G$ such that $\overline{\mathscr{B}}_{G} \sim \overline{\mathscr{B}}_{W}$. (ii) If $\overline{\mathscr{B}}_{G}$ is any basic set for $G$ then there exists a basic set $\overline{\mathscr{B}}_{W}$ for $W$ such that $\overline{\mathscr{B}}_{G} \sim \overline{\mathscr{B}}_{W}$.

Proof. (i) We apply (2.22) to both $G$ and $W$. To indicate the dependence on $G$ and $W$ we write $c_{G}, \mathbf{E}_{G}, \mathbf{M}_{G}, \mathbf{N}_{G}$ and $c_{W}, \mathbf{E}_{W}, \mathbf{M}_{W}, \mathbf{N}_{W}$. Define polynomials $p_{j}$ by $d_{j}^{W} \bar{f}_{j}^{W}=p_{j}\left(d_{1}^{W} f_{1}^{W}, \cdots, d_{l}^{W} f_{l}^{W}\right)$. Define $\bar{f}_{j}^{G}$ by $d_{j}^{G} \bar{f}_{j}^{G}$ $=p_{j}\left(d_{1}^{G} f_{1}^{G}, \cdots, d_{l}^{G} f_{l}^{G}\right)$. Then $c_{G}=c_{W}$ and $\mathbf{M}_{G}=\mathbf{M}_{W}$. It follows from (1.6) that $\mathbf{E}_{G}=k \cdot \mathbf{E}_{W}$ for some $k \in C^{*}$. Thus $\mathbf{N}_{G}=\mathbf{N}_{W}$. Assertion (i) follows from (2.23) applied to both $G$ and $W$ and the assumption $\mathbf{D}_{G}\left(T_{1}, \cdots, T_{l} ; \mathscr{B}_{G}\right)$ $=\mathbf{D}_{W}\left(T_{1}, \cdots, T_{l} ; \mathscr{B}_{W}\right)$. Assertion (ii) is proved in the same way.

\section{§3. The case $\operatorname{dim} V=2$}

(3.1) Theorem. Suppose $\operatorname{dim} V=2$ and $G \subset G L(V)$ is a Shephard group with symbol $p_{1}[q] p_{2}$. Then there exists a basic set $\mathscr{B}=\left\{f_{1}, f_{2}\right\}$ such that

$$
\mathbf{D}\left(T_{1}, T_{2} ; \mathscr{B}\right)=\left[\begin{array}{ll}
T_{1} & T_{2} \\
T_{2} & T_{1}^{q-1}
\end{array}\right] .
$$

Theorem (3.1) is a consequence of Lemmas (3.4)-(3.9). We introduce some notation. If $p_{1}, p_{2} \in S$ we write $J\left(p_{1}, p_{2}\right)=\operatorname{det} \mathbf{J}\left(p_{1}, p_{2}\right)$ for the Jacobian determinant, a notation used throughout this section for various polynomials $p_{1}, p_{2}$. It follows from (2.5) that for any basic set $\mathscr{B}=\left\{f_{1}, f_{2}\right\}$ we have $J\left(f_{1}, f_{2}\right) \approx J$. Let $\theta_{1}, \theta_{2}$ be the basic derivations associated to $\mathscr{B}$ as in (2.18). Since $\theta_{1}=x_{1} D_{1}+x_{2} D_{2}$ is the Euler derivation, (2.4) and (2.19. iii) give

$$
\Delta\left(f_{1}, f_{2} ; \mathscr{B}\right)=\left[\begin{array}{ll}
d_{1} f_{1} & d_{2} f_{2} \\
d_{2} f_{2} & \theta_{2} f_{2}
\end{array}\right] .
$$

Let $\psi\left(T_{1}, T_{2}\right)$ be the polynomial defined by $\psi\left(d_{1} f_{1}, d_{2} f_{2}\right)=\theta_{2} f_{2}$. Thus

$$
\mathbf{D}\left(T_{1}, T_{2} ; \mathscr{B}\right)=\left[\begin{array}{cc}
T_{1} & T_{2} \\
T_{2} & \psi\left(T_{1}, T_{2}\right)
\end{array}\right] .
$$

To prove (3.1) it remains to show that 


$$
\theta_{2} f_{2}=\left(d_{1} f_{1}\right)^{q-1}
$$

for suitable choice of $f_{1}, f_{2}$. Then $\psi\left(T_{1}, T_{2}\right)=T_{1}^{q-1}$.

(3.4) Lemma. $\quad \theta_{2} f_{2} \neq 0$.

Proof. Suppose $\theta_{2} f_{2}=0$. It follows from (2.6) and (3.2) that $J Q \approx f_{2}^{2}$. Since $Q$ is square-free, $Q$ divides $f_{2}$, and thus $f_{2}$ divides $J$. For $g \in G$ let $\delta(g)=\operatorname{det} g$. It is known [21, p. 85] that $J$ is a semi-invariant of $G$ of minimal degree with character $\delta$. Since $J / f_{2}$ is also a semi-invariant of $G$ with character $\delta$ we have a contradiction.

We have already remarked preceding (2.22) that, since $G$ is a Shephard group, the invariant $f_{1}$ is unique up to a constant multiple. We showed in [14] that $\left\{f_{1}, J\left(Q, f_{1}\right)\right\}$ is a basic set. Suppose $\left\{f_{1}, f_{2}\right\}$ is any basic set. Then $f_{2} \in \sum C f_{1}^{\alpha} J\left(Q, f_{1}\right)^{\beta}$ where the sum is over all nonnegative integers $\alpha, \beta$ such that $\alpha d_{1}+\beta d_{2}=d_{2}$. Thus either $(\alpha, \beta)=(0,1)$ or $\beta=0$, in which case $d_{1}$ divides $d_{2}$. It follows from (2.13) that $2 d_{2}=q d_{1}$ so $\beta=0$ can only occur if $q$ is even. Thus there are two possibilities:

$$
\begin{array}{ll}
f_{2}=a J\left(Q, f_{1}\right) & \text { if } q \text { is odd, } \\
f_{2}=a J\left(Q, f_{1}\right)+b f_{1}^{r} & \text { if } q=2 r \text { is even, }
\end{array}
$$

where $a \in C^{*}$ and $b \in C$. In the next lemma we compute the basic derivations $\theta_{1}, \theta_{2}$ associated to $f_{1}, f_{2}$ where $f_{2}$ is given by (3.5). Let

$$
\eta=-\left(D_{2} Q\right) D_{1}+\left(D_{1} Q\right) D_{2} .
$$

Note that $r \varphi=J(Q, \varphi)$ for any $\varphi \in S$.

(3.7) Lemma. Let $G$ be a Shephard group. Let $f_{1}$ be an invariant form of degree $d_{1}$ and let $f_{2}$ be defined by (3.5). Then the basic derivations $\theta_{1}, \theta_{2}$ associated to the basic invariants $f_{1}, f_{2}$ are $\theta_{1}=x_{1} D_{1}+x_{2} D_{2}$ and

(i) $\theta_{2}=d_{2} a \eta \quad$ if $q$ is odd,

(ii) $\theta_{2}=d_{2} a \eta+b r f_{1}^{r-1} \theta_{1}$ if $q=2 r$ is even.

Proof. Recall from (2.5) that $n=\operatorname{deg} Q$. We showed in [14] that $\mathbf{H}\left(f_{1}\right) \mathbf{Q}\left(\theta_{1}, \eta\right)=m_{1} \mathbf{J}\left(f_{1}, \psi\right)$ where $\left(n+d_{1}-2\right) \psi=J\left(Q, f_{1}\right)$. Note that $n+d_{1}$ $-2=\operatorname{deg} J\left(Q, f_{1}\right)=\operatorname{deg} \psi=d_{2}$. In view of the equivalence of (2.16) and (2.17) we have $\operatorname{Hess}\left(f_{1}\right) \theta_{1}=m_{1} d f_{1}$ and $\operatorname{Hess}\left(f_{1}\right) \eta=m_{1} d \psi$. Now $R$-linearity of Hess $\left(f_{1}\right)$ shows that $\operatorname{Hess}\left(f_{1}\right) \theta_{2}=m_{1} d f_{2}$. 
(3.8) Lemma. The form $J\left(Q, J\left(Q, f_{1}\right)\right)$ is a nonzero invariant of degree $2 d_{2}-d_{1}=(q-1) d_{1}$.

Proof. Let $f, \varphi, \psi$ be any binary forms of degrees $m, n, p$ at least 2 . Let $H(f)=\operatorname{det} \mathbf{H}(f)$ be the Hessian determinant of $f$ and let

$$
\tau_{2}(f, \varphi)=\left(D_{1}^{2} f\right)\left(D_{2}^{2} \varphi\right)-2\left(D_{1} D_{2} f\right)\left(D_{1} D_{2} \varphi\right)+\left(D_{2}^{2} f\right)\left(D_{1}^{2} \varphi\right)
$$

be their second transvectant [21, p. 57]. Define $(f, \varphi)^{2}$ by

$$
\tau_{2}(f, \varphi)=m(m-1) n(n-1)(f, \varphi)^{2} .
$$

We use a known formula for the Jacobian of a Jacobian [23, p. 223]:

$$
J(J(f, \varphi), \psi) \approx \frac{m-n}{m+n-2}(f, \varphi)^{2} \psi+(f, \psi)^{2} \varphi-(\varphi, \psi)^{2} f .
$$

Set $f=f_{1}$ and $\varphi=\psi=Q$. We proved in [14] that $\tau_{2}(f, Q)=0$. Thus

$$
J\left(Q, J\left(Q, f_{1}\right)\right) \approx \tau_{2}(Q, Q) f_{1} \approx H(Q) f_{1} .
$$

Since $Q$ is not a power of a linear form $H(Q) \neq 0[6$, p. 235] and thus $J\left(Q, J\left(Q, f_{1}\right)\right) \neq 0$. In general, if $G$ is a subgroup of $G L(V)$ and $f, \varphi$ are semi-invariants of $G$ with characters $\lambda, \mu$ then $J(f, \varphi)$ is a semi-invariant of $G$ with character $\delta \lambda \mu[21$, p. 97] where $\delta(g)=\operatorname{det} g$. Since $Q$ is a semi-invariant with character $\delta^{-1}[14,2.27]$ and $f_{1}$ is an invariant form it follows that $J\left(Q, J\left(Q, f_{1}\right)\right)$ is an invariant of degree $2 n+d_{1}-4$. We remarked in the proof of (3.7) that $n+d_{1}-2=d_{2}$. Since $2 d_{2}=q d_{1}$ the proof is complete.

(3.9) Lemma. (i) If $q$ is odd then there exists $c \in C^{*}$ with $J\left(Q, J\left(Q, f_{1}\right)\right)=c f_{1}^{q-1}$. (ii) If $q=2 r$ is even then there exist $c_{1}, c_{2} \in C$ not both zero with $J\left(Q, J\left(Q, f_{1}\right)\right)=c_{1} f_{1}^{r-1} J\left(Q, f_{1}\right)+c_{2} f_{1}^{q-1}$.

Proof. It follows from (3.8) that $J\left(Q, J\left(Q, f_{1}\right)\right) \in \sum C f_{1}^{\alpha} f_{2}^{\beta}$ where the sum is over all nonnegative integers $\alpha, \beta$ such that $\alpha d_{1}+\beta d_{2}=2 d_{2}-d_{1}$. Thus $\beta=0,1$. If $\beta=0$ then $\alpha d_{1}=2 d_{2}-d_{1}$. Since $2 d_{2}=q d_{1}$ it follows that $\alpha=q-1$. If $\beta=1$ then $(\alpha+1) d_{1}=d_{2}$ so $d_{1}$ divides $d_{2}$ and $q$ must be even. Since $J\left(Q, J\left(Q, f_{1}\right)\right) \neq 0$ this completes the proof.

To prove (3.3) it remains to show that constants $a \in C^{*}$ and $b \in C$ may be chosen so that $\theta_{2} f_{2}=\left(d_{1} f_{1}\right)^{q-1}$. Suppose first that $q$ is odd. Then by (3.5)-(3.9) we have 


$$
\theta_{2} f_{2}=d_{2} a^{2} \eta J\left(Q, f_{1}\right)=d_{2} a^{2} J\left(Q, J\left(Q, f_{1}\right)\right)=d_{2} a^{2} c f_{1}^{q-1} .
$$

Since $c \neq 0$ we may choose $a \in C^{*}$ so that $d_{2} a^{2} c=d_{1}^{q-1}$. Now suppose $q=2 r$ is even. A degree argument as in (3.9) shows that $\theta_{2} f_{2}=k_{1} f_{1}^{r-1} J\left(Q, f_{1}\right)$ $+k_{2} f_{1}^{q-1}$ where $k_{1}, k_{2} \in C$. It follows from (3.3) that $k_{1}, k_{2}$ are not both zero. We must show that there exist $a \in C^{*}, b \in C$ such that $k_{1}=0$ and $k_{2}=d_{1}^{q-1}$. Direct calculation gives

$$
\begin{aligned}
\theta_{2} f_{2} & =d_{2} a^{2} \eta J\left(Q, f_{1}\right)+d_{2} a b \eta f_{1}^{r}+b r f_{1}^{r-1} \theta_{1} f_{2} \\
& =d_{2} a^{2} J\left(Q, J\left(Q, f_{1}\right)\right)+2 d_{2} a b r f_{1}^{r-1} J\left(Q, f_{1}\right)+d_{2} b^{2} r f_{1}^{q-1} .
\end{aligned}
$$

From (3.9) we get $k_{1}=d_{2} a^{2} c_{1}+2 d_{2} a b r$ and $k_{2}=d_{2} a^{2} c_{2}+d_{2} b^{2} r$. Choose $b \in C$ so that $a c_{1}+2 b r=0$. Then $k_{1}=0$ and $k_{2}=a^{2}\left(d_{2} c_{2}+d_{2} c_{1}^{2} / 4 r\right)$. Since $k_{1}=0$ we have $k_{2} \neq 0$ and thus we may choose $a \in C^{*}$ so that $k_{2}=d_{1}^{q-1}$. This proves (3.3) and hence completes the argument for (3.1).

(3.10) Remark. It follows from the preceding computation that for given $f_{1}$, an invariant $f_{2}$ which satisfies (3.1) is determined uniquely up to sign.

To complete the proof of Theorem (2.25) in case $\operatorname{dim} V=2$, we apply (3.1) to both $G$ and $W$, where $W$ has symbol $2[q] 2$, to conclude that there exist bases $\mathscr{B}_{G}, \mathscr{B}_{W}$ such that

$$
\mathbf{D}_{G}\left(T_{1}, T_{2} ; \mathscr{B}_{G}\right)=\left[\begin{array}{ll}
T_{1} & T_{2} \\
T_{2} & T_{1}^{q-1}
\end{array}\right]=\mathbf{D}_{W}\left(T_{1}, T_{2} ; \mathscr{B}_{W}\right)
$$

Table 2.

\begin{tabular}{clll}
\hline $\mathrm{S} \& \mathrm{~T}$ & Coxeter & $f_{1}$ & \multicolumn{1}{c}{$f_{2}$} \\
\hline$G(q, q ; 2)$ & $2[q] 2$ & $x_{1} x_{2}$ & $\left(2^{q / 2-1} / q\right)\left(x_{1}^{q}+x_{2}^{q}\right)$ \\
$G(p, 1 ; 2)$ & $p[4] 2$ & $x_{1}^{p}+x_{2}^{p}$ & $(p / 2)\left(x_{1}^{2 p}-6 x_{1}^{p} x_{2}^{p}+x_{2}^{2 p}\right)$ \\
4 & $3[3] 3$ & $\Phi$ & $8(-1 / 3)^{1 / 4} t$ \\
8 & $4[3] 4$ & $W$ & $(4 / 3) 2^{1 / 2} \chi$ \\
16 & $5[3] 5$ & $H$ & $(4 / 3)(-5)^{1 / 2} T$ \\
5 & $3[4] 3$ & $t$ & $(1 / 6)(-3)^{1 / 2} \chi$ \\
10 & $4[4] 3$ & $\chi$ & $6\left(2 W^{3}-\chi^{2}\right)$ \\
18 & $5[4] 3$ & $T$ & $15\left(T^{2}+2 H^{3}\right)$ \\
20 & $3[5] 3$ & $f$ & $(2 / 5) T$ \\
6 & $3[6] 2$ & $\Phi$ & $(16 / 3)\left(\Phi^{3}-2 \Psi^{3}\right)$ \\
9 & $4[6] 2$ & $W$ & $(64 / 3)\left(W^{3}-2 \chi^{2}\right)$ \\
17 & $5[6] 2$ & $H$ & $(400 / 3)\left(2 T^{2}+H^{3}\right)$ \\
14 & $3[8] 2$ & $t$ & $(1 / 2)\left(\chi^{2}+W^{3}\right)$ \\
21 & $3[10] 2$ & $f$ & $(12 / 5)\left(H^{3}-T^{2}\right)$ \\
\hline
\end{tabular}


In Table 2 we list the Shephard groups for $l=2$ together with a basic set $\mathscr{B}=\left\{f_{1}, f_{2}\right\}$ which satisfies Theorem (3.1). The invariant $f_{1}$ is chosen as in $[19,4.14]$ to be one of Klein's polynomials $\Phi, \Psi, t, W, \chi, f$, $H, T$, [7]. The invariant $f_{2}$, determined up to sign, is also expressed in terms of Klein's polynomials.

\section{§4. The case $\operatorname{dim} V \geq 3$}

If $\operatorname{dim} V \geq 3$ the Shephard groups which are not Coxeter groups are the monomial groups $G(p, 1, l)$ and the groups $G_{25}, G_{26}, G_{32}$ in the classification of Shephard and Todd. We use (2.27) which tells us that it suffices to exhibit basic sets $\mathscr{B}_{G}, \mathscr{B}_{W}$ such that $\mathbf{D}_{G}\left(T_{1}, \ldots, T_{l} ; \mathscr{B}_{G}\right)=\mathbf{D}_{W}\left(T_{1}, \cdots\right.$, $\left.T_{l} ; \mathscr{B}_{W}\right)$.

First we consider the monomial group $G=G(p, 1, l)$. In this case the corresponding Coxeter group $W=G(2,1, l)$ is of type $B_{l}$. It suffices to show that $G$ has a basic set $\mathscr{B}$ such that the corresponding discriminant matrix $\mathbf{D}\left(T_{1}, \cdots, T_{l} ; \mathscr{B}\right)$ is the same for all $p$. For $k=1,2,3, \cdots$ let $s_{k}=x_{1}^{k}+\cdots+x_{l}^{k}$. Define polynomials $\varphi_{k}=\varphi_{k}\left(T_{1}, \cdots, T_{l}\right)$ by

$$
s_{k}=\varphi_{k}\left(s_{1}, \cdots, s_{l}\right) \quad k=1,2,3, \cdots \text {. }
$$

Thus $\varphi_{k}=T_{k}$ for $k=1, \cdots, l$. Let $\mathscr{B}=\left\{f_{1}, \cdots, f_{l}\right\}$ be the basic set for $G(p, 1, l)$ defined by

$$
k p f_{k}=s_{k p} \quad 1 \leq k \leq l .
$$

It follows from (2.17) that the associated basic derivations are

$$
\theta_{k}=\sum_{j=1}^{l} x_{j}^{(k-1) p+1} D_{j} \quad 1 \leq k \leq l
$$

Thus

$$
\mathbf{J}\left(f_{1}, \cdots, f_{l}\right)^{T} \mathbf{Q}\left(\theta_{1}, \cdots, \theta_{l}\right)=\left(\begin{array}{cccc}
s_{p} & s_{2 p} & \cdots & s_{l p} \\
s_{2 p} & s_{3 p} & \cdots & s_{(l+1) p} \\
\vdots & \vdots & \vdots & \vdots \\
s_{l p} & s_{(l+1) p} & \cdots & s_{(2 l-1) p}
\end{array}\right) .
$$

Since $s_{k p}=\varphi_{k}\left(s_{p}, \cdots, s_{l p}\right)$, the discriminant matrix

$$
\mathbf{D}\left(T_{1}, \cdots, T_{l} ; \mathscr{B}\right)=\left(\begin{array}{cccc}
\varphi_{1} & \varphi_{2} & \cdots & p_{l} \\
\varphi_{2} & \varphi_{3} & \cdots & p_{l+1} \\
\vdots & \vdots & \vdots & \vdots \\
p_{l} & \varphi_{l+1} & \cdots & \varphi_{2 l-1}
\end{array}\right)
$$


is the same for all $p$. This proves (2.25) for $G=G(p, 1, l)$ and $W=B_{l}$.

To complete the proof of the main theorem we have to find $\mathscr{B}_{G}, \mathscr{B}_{W}$ such that $\mathscr{B}_{G} \sim \mathscr{B}_{W}$ for the three remaining pairs $(G, W)$ which are $\left(G_{25}, D_{3}\right),\left(G_{26}, B_{3}\right)$ and $\left(G_{32}, A_{4}\right)$. There are done by explicit calculation, in part using machine computation in MACSYMA. We choose a basic set $\mathscr{B}_{W}=\left\{f_{1}^{W}, \cdots, f_{l}^{W}\right\}$. Since $f_{1}^{W}$ is a quadratic form, the Hessian $\mathbf{H}\left(f_{1}^{W}\right)$ is a constant matrix, so it is easy to compute the matrix $\mathbf{Q}\left(\theta_{1}^{W}, \cdots, \theta_{l}^{W}\right)=$ $m_{1}^{W} \mathbf{H}\left(f_{1}^{W}\right)^{-1} \mathbf{J}\left(f_{1}^{W}, \cdots, f_{l}^{W}\right)$ which satisfies (2.17). Note that $m_{1}^{W}=1$. Next we compute $\mathbf{J}\left(f_{1}^{W}, \cdots, f_{l}^{W}\right)^{T} \mathbf{Q}\left(\theta_{1}^{W}, \cdots, \theta_{l}^{W}\right)$. This is a matrix of $W$-invariants. We express each matrix entry as a polynomial in the variables $d_{1}^{W} f_{1}^{W}$, $\cdots, d_{l}^{W} f_{l}^{W}$ to obtain the matrix $\mathbf{D}_{W}\left(T_{1}, \cdots, T_{l} ; \mathscr{B}_{W}\right)$. Basic invariants for the corresponding groups $G$ were determined by Shephard and Todd using work of Maschke [9]. Let $\mathscr{B}=\left\{f_{1}, \cdots, f_{l}\right\}$ be their basic set for $G$. Any basic invariant $f_{i}^{G}$ of degree $d_{\imath}^{G}$ has the form $f_{i}^{G}=\varphi_{i}\left(f_{1}, \cdots, f_{l}\right)$ where the $\varphi_{i}$ are polynomials. Since the $G$-invariant form of minimal degree is unique up to a constant we may choose $f_{1}^{G}=f_{1}$. Degree considerations restrict the polynomials $\varphi_{i}$ so that there are only a few free parameters in each case. The matrix $\mathbf{J}\left(f_{1}^{G}, \cdots, f_{l}^{G}\right)$ contains these parameters. The matrix $\mathbf{H}\left(f_{1}^{G}\right)$ has polynomial entries. In $G_{32}$ the entries of the $4 \times 4$ matrix $\mathbf{H}\left(f_{1}^{G}\right)$ are polynomials of degree 10 in 4 variables. Thus it is not easy to compute the matrix $\mathbf{Q}\left(\theta_{1}^{G}, \cdots, \theta_{l}^{G}\right)=m_{1}^{G} \mathbf{H}\left(f_{1}^{G}\right)^{-1} \mathbf{J}\left(f_{1}^{G}, \cdots, f_{l}^{G}\right)$ which satisfies (2.17). As in the case of $W$ we compute $\mathbf{J}\left(f_{1}^{G}, \cdots, f_{l}^{G}\right)^{T} \mathbf{Q}\left(\theta_{1}^{G}, \cdots, \theta_{l}^{G}\right)$ which still contains the free parameters. This is a matrix of $G$-invariants. We express each matrix entry as a polynomial in the variables $d_{1}^{G} f_{1}^{G}, \cdots, d_{l}^{G} f_{l}^{G}$ and force the parameters to satisfy $\mathbf{D}_{G}\left(T_{1}, \cdots, T_{l} ; \mathscr{B}_{G}\right)=\mathbf{D}_{W}\left(T_{1}, \cdots, T_{l} ; \mathscr{B}_{W}\right)$. The results of the calculation are given in the Appendix where we also exhibit the unique basic derivations $\theta_{1}, \cdots, \theta_{l}$ associated to $\mathscr{B}_{G}$ which satisfy (2.17).

\section{$\S 5$. Related results}

The comparison theorem (2.25) allows us to deduce further properties of a Shephard group $G$ from the corresponding properties of the associated Coxeter group $W$.

(5.1) Theorem. If $G \subset G L(V)$ is a Shephard group then $M(G)=V$ - $\bigcup_{H \in \mathscr{A}(G)} H$ is a $K(\pi, 1)$ space.

Proof. Let $W$ be the Coxeter group associated to $G$. Deligne [5] 
showed that $M(W)$ is a $K(\pi, 1)$ space and hence so is $M(W) / W$. It follows from (1.7) that for suitable choice of basic invariants the discriminant loci for $G$ and $W$ are the zero sets of the same polynomial. Thus $M(G) / G=M(W) / W$, so $M(G) / G$ and hence $M(G)$ is a $K(\pi, 1)$ space.

If $l=2$ then the complement of any finite set of hyperplanes containing the origin is a $K(\pi, 1)$ space. We proved in [13] that $M(G)$ is a $K(\pi, 1)$ space for the Shephard groups $G=G(p, 1, l)$. Nakamura [11] showed that $M(G)$ is a $K(\pi, 1)$ space for all imprimitive unitary reflection groups.

(5.2) Theorem. Let $G \subset G L(V)$ be a Shephard group and let $\mathscr{B}_{G}=$ $\left\{f_{1}^{G}, \cdots, f_{l}^{G}\right\}$ be a basic set. Then

$$
\Delta_{G}\left(f_{1}^{G}, \cdots, f_{l}^{G}\right) \equiv\left(f_{l}^{G}\right)^{l} \quad \bmod \left(f_{1}^{G}, \cdots, f_{l-1}^{G}\right)
$$

Proof. Let $W$ be the associated Coxeter group. By (2.25) there exists a basic set $\mathscr{B}_{W}=\left\{f_{1}^{W}, \cdots, f_{l}^{W}\right\}$ such that $\mathscr{B}_{G} \sim \mathscr{B}_{W}$. Saito [15] proved that $\Delta_{W}\left(f_{1}^{W}, \cdots, f_{l}^{W} ; \mathscr{B}_{W}\right) \equiv\left(f_{l}^{W}\right)^{l} \bmod \left(f_{1}^{W}, \cdots, f_{l-1}^{W}\right)$. Thus $\Delta_{W}\left(T_{1}, \cdots, T_{l} ; \mathscr{B}_{W}\right) \equiv$ $T_{l}^{\iota} \bmod \left(T_{1}, \cdots, T_{l-1}\right)$. The assertion follows from (2.26).

Suppose $W \subset G L(V)$ is a finite irreducible Coxeter group. Let $\mathscr{B}=\left\{f_{1}, \cdots, f_{l}\right\}$ be a basic set for $W$ and write $\mathbf{J}=\mathbf{J}\left(f_{1}, \cdots, f_{l}\right)$. Saito, Sekiguchi and Yano $[15,16,24]$ have used the matrix $\mathbf{J}^{T} \mathbf{J}$. We may choose coordinates $x_{1}, \cdots, x_{i}$ so that $f_{1}=\sum x_{i}^{2}$ and choose $\theta_{j}=\frac{1}{2} \sum\left(D_{i} f_{j}\right) D_{i}$. Then $\mathbf{J}=2 \mathbf{Q}\left(\theta_{1}, \cdots, \theta_{l}\right)$ and (2.17) is satisfied. Thus $\mathbf{J}^{T} \mathbf{J} \approx \boldsymbol{\Delta}\left(f_{1}, \cdots, f_{l} ; \mathscr{B}\right)$ in the notation of this paper. Since $d_{l-1}<d_{l}$ the operator $\partial / \partial f_{l}: R \rightarrow R$ is uniquely determined up to constant. Saito, Sekiguchi and Yano $[15,16]$ proved that there exists a basic set $\mathscr{B}$ for $W$, which they call a flat basic set, such that $\left(\partial / \partial f_{l}\right)\left(\mathbf{J}^{T} \mathbf{J}\right) \in M_{l}(\boldsymbol{R})$.

If $G \subset G L(V)$ is a Shephard group, it follows from (1.6) that $d_{l-1}^{G}<d_{l}^{G}$ and thus the operator $\partial / \partial f_{l}: R \rightarrow R$ is again uniquely determined up to constant.

(5.3) Definition. Let $G$ be a Shephard group. We call a basic set $\mathscr{B}$ a flat basic set if $\left(\partial / \partial f_{l}\right) \Delta\left(f_{1}, \cdots, f_{l} ; \mathscr{B}\right) \in M_{l}(C)$.

(5.4) Theorem. Let $G \subset G L(V)$ be a Shephard group. Then $G$ has a flat basic set.

Proof. In view of (2.20) a basic set $\mathscr{B}$ is flat if and only if 
$\left(\partial / \partial T_{l}\right) \mathbf{D}\left(T_{1}, \cdots, T_{l} ; \mathscr{B}\right) \in M_{l}(C)$. The result follows from $(2.25)$ and the existence of flat basic sets for finite irreducible Coxeter groups.

(5.5) Remark. If $\operatorname{dim} V=2$ then

$$
\frac{\partial}{\partial T_{2}}\left[\begin{array}{ll}
T_{1} & T_{2} \\
T_{2} & T_{1}^{q-1}
\end{array}\right]=\left[\begin{array}{ll}
0 & 1 \\
1 & 0
\end{array}\right] .
$$

Thus the basic sets constructed in Theorem (3.1) are flat.

We conclude this section with some results about the discriminant which are true for any irreducible unitary reflection group $G \subset G L(V)$. Let $\mathscr{B}=\left\{f_{1}, \cdots, f_{l}\right\}$ be a basic set and let $\mathscr{D}=\left\{\theta_{1}, \cdots, \theta_{l}\right\}$ be a set of basic derivations. As before let $R=S^{a}=C\left[f_{1}, \cdots, f_{l}\right]$. Let $\operatorname{Der}_{R}$ be the $R$-module of derivations of $R$. Then $\operatorname{Der}_{R}$ has an $R$-basis $\left\{D_{f_{i}}=\partial / \partial f_{i}\right\}$ where $D_{f_{i}}\left(f_{j}\right)=\delta_{i j}$. If $\theta \in \operatorname{Der}_{S}$ and $a \in S$ then $g(a \theta)=(g a)(g \theta)$ for all $g \in G$. Thus if $\theta \in \operatorname{Der}_{S}^{G}$ then $\theta R \subseteq R$. Let $\bar{\theta}$ be the restriction of $\theta$ to $R$. Then

$$
\bar{\theta}=\sum_{i=1}^{l} \theta\left(f_{i}\right) D_{f_{i}} .
$$

As in the introduction let $\mathscr{A}$ be the set of reflecting hyperplanes and let $\Delta=\Delta\left(f_{1}, \cdots, f_{l} ; \mathscr{B}\right)$ be the discriminant. The following proposition is due to H. Terao; see [22, Thm. D] for the analytic version.

(5.7) Proposition. Let $D_{R}(\Delta)=\left\{\eta \in \operatorname{Der}_{R} \mid \eta \Delta \in R \Delta\right\}$. If $\theta_{1}, \cdots, \theta_{l}$ is an $R$-basis for $\operatorname{Der}_{S}^{G}$ then $D_{R}(\Delta)$ is a free $R$-module with $R$-basis $\bar{\theta}_{1}, \cdots, \bar{\theta}_{l}$.

(5.8) Remark. In view of (5.6) and (2.2) the columns of the matrix $\Delta\left(f_{1}, \cdots, f_{l} ; \mathscr{B}, \mathscr{D}\right)$ defined in $(2.4)$ are the coefficients of the derivations $\bar{\theta}_{1}, \cdots, \bar{\theta}_{l}$ when written as $R$-linear combinations of $D_{f_{1}}, \cdots, D_{f_{l}}$.

We have used (1.6) in the proof of the comparison theorem and hence in the proof of (1.7). We show in (5.15) that conversely (1.7) implies (1.6). To do this we must consider gradings of $R=C\left[f_{1}, \cdots, f_{l}\right]$. There is a natural grading $R=\oplus R_{p}$ inherited from $S$ in which $\operatorname{deg} f_{i}=d_{i}$ and $R_{p}$ $=R \cap S_{p}$. If $\alpha=\left(a_{1}, \cdots, a_{l}\right)$ is any $l$-tuple of positive integers we may also grade $R$ by letting $\operatorname{deg} f_{i}=a_{i}$. Let $R_{p}^{\alpha}$ denote the $p$-th homogeneous component in this grading. Thus $R=\oplus R_{p}^{\alpha}$. If $f \in R_{p}^{\alpha}$ we say that $f$ is $\left(a_{1}, \cdots, a_{l}\right)$-homogeneous of degree $p$. If $f \in R_{p}^{\alpha}$ then the Euler formula says

$$
\sum_{i=1}^{l} a_{i} f_{i} D_{f_{i}}(f)=p f
$$


Let $\theta \in \operatorname{Der}_{R}$. We say that $\theta$ is $\left(a_{1}, \cdots, a_{l}\right)$-homogeneous of degree $r$ if $\theta\left(R_{p}^{\alpha}\right) \subseteq R_{p+r}^{\alpha}$. For example $D_{f_{i}}$ is $\left(a_{1}, \cdots, a_{l}\right)$-homogeneous of degree $-a_{i}$. It follows from (2.5) and (2.6) that in the natural grading the discriminant $\Delta$ is $\left(d_{1}, \cdots, d_{l}\right)$-homogeneous of degree $m+n$.

(5.10) Proposition. Let $G \subset G L(V)$ be any finite irreducible unitary reflection group. Let $\left(a_{1}, \cdots, a_{l}\right)$ be an l-tuple of positive integers. Suppose $\Delta$ is $\left(a_{1}, \cdots, a_{l}\right)$-homogeneous of degree $p$. Then there exists $k$ such that $p=k(m+n)$ and $a_{i}=k d_{i}$ for $1 \leq i \leq l$.

Proof. Let $\theta_{1}, \cdots, \theta_{l}$ be a set of basic derivations of degrees $n_{1}-1$, $\cdots, n_{l}-1$. It follows from (5.7) that $\bar{\theta}_{1}, \cdots, \bar{\theta}_{l}$ is an $R$-basis for $D_{R}(\Delta)$. Note that $\bar{\theta}_{i}$ is $\left(d_{1}, \cdots, d_{l}\right)$-homogeneous of degree $n_{i}-1$. We proved in [12] that $1=n_{1}<n_{2}$ for any irreducible unitary reflection group. Thus

$$
\bar{\theta}_{1}=d_{1} f_{1} D_{f_{1}}+\cdots+d_{l} f_{l} D_{f_{l}}
$$

is, up to constant, the unique $\left(d_{1}, \cdots, d_{l}\right)$-homogeneous element of degree 0 in $D_{R}(\Delta)$. Let $\left(a_{1}, \cdots, a_{l}\right)$ be any $l$-tuple of positive integers such that $\Delta$ is $\left(a_{1}, \cdots, a_{l}\right)$-homogeneous of degree $p$. Define

$$
\eta=a_{1} f_{1} D_{f_{1}}+\cdots+a_{l} f_{l} D_{f_{l}} .
$$

By the Euler formula (5.9) we have $\eta \Delta=p \Delta$ so $\eta \in D_{R}(\Delta)$. Clearly $\eta$ is $\left(d_{1}, \cdots, d_{l}\right)$-homogeneous of degree 0 . Thus there exists $k \neq 0$ such that $\eta=k \bar{\theta}_{1}$. The conclusion follows.

Proposition (5.10) may be restated using Milnor's notion [10, p. 75] of weighted homogeneous polynomials. In the $\left(d_{1}, \cdots, d_{l}\right)$-grading of $C\left[T_{1}, \cdots, T_{l}\right]$ the polynomial $\Delta=\Delta\left(T_{1}, \cdots, T_{l} ; \mathscr{B}\right)$ is homogeneous of degree $(m+n)$ and hence it is weighted homogeneous with weights $\left((m+n) / d_{1}\right.$, $\left.\cdots,(m+n) / d_{l}\right)$.

(5.13) Corollary. Let $G \subset G L(V)$ be any irreducible unitary reflection group. The discriminant $\Delta\left(T_{1}, \cdots, T_{l} ; \mathscr{B}\right)$ has uniquely determined weights. These weights are also independent of $\mathscr{B}$.

(5.14) Remark. The assertions in (5.10) and (5.13) need not hold for reducible groups. For example if $G$ is of type $A_{1} \times A_{1}$ acting naturally on $C^{2}$ then $Q=x_{1} x_{2}$. If we choose $\mathscr{B}=\left\{x_{1}^{2}, x_{2}^{2}\right\}$ then $\Delta\left(T_{1}, T_{2} ; \mathscr{B}\right)=T_{1} T_{2}$, which is $\left(a_{1}, a_{2}\right)$-homogeneous of degree $a_{1}+a_{2}$ for any positive integers $a_{1}, a_{2}$. The weights are $\left(\left(a_{1}+a_{2}\right) / a_{1},\left(a_{1}+a_{2}\right) / a_{2}\right)$. 
(5.15) Corollary. Let $G \subset G L(V)$ be a Shephard group and let $W$ be the corresponding Coxeter group. Suppose there exist basic sets $\mathscr{B}_{G}$, $\mathscr{B}_{W}$ such that $\Delta_{G}\left(T_{1}, \cdots, T_{l} ; \mathscr{B}_{G}\right)=\Delta_{W}\left(T_{1}, \cdots, T_{l} ; \mathscr{B}_{W}\right)$. Then $d_{1}^{G} / d_{1}^{W}=\cdots$ $=d_{l}^{G} / d_{l}^{W}$.

Proof. Apply (5.13) to both $\Delta_{G}\left(T_{1}, \cdots, T_{l} ; \mathscr{B}_{G}\right)$ and $\Delta_{W}\left(T_{1}, \cdots, T_{l} ; \mathscr{B}_{W}\right)$, This shows $\left(m^{G}+n^{G}\right) / d_{i}^{G}=\left(m^{W}+n^{W}\right) / d_{i}^{W}$ for $1 \leq i \leq l$.

\section{Appendix}

(A.1) The pairs $(G, W)$. In Table 1 we list the pairs $(G, W)$ where $G$ is a Shephard group, $W$ is the corresponding Coxeter group and $G \neq W$. Given the graph $\Gamma(G)$ we obtain $\Gamma(W)$ by omitting the labels on the nodes of $\Gamma(G)$.

(A.2) Flat basic sets for $l=2$. In Table 2 we list basic sets $\mathscr{B}=$ $\left\{f_{1}, f_{2}\right\}$ for Shephard groups $G$ with $l=2$ which satisfy (3.1). It follows from (5.5) that these basic sets are flat. The invariants are either given explicitly or in terms of the polynomials $\Phi, \Psi, t, W, \chi, f, H, T$ of Klein [7].

(A.3) Conventions. For the Shephard groups $G_{25}, G_{26}, G_{32}$ we use work of Maschke [9]. The computations were done using MACSYMA. In order to be able to use the polynomials $C_{6}, C_{9}, C_{12}, C_{18}$ defined in [9, p. 326] and $F_{12}, F_{18}, F_{24}, F_{30}$ defined in [9, p. 337] we agree to let the basis of $V^{*}$ be $z_{1}, z_{2}, z_{3}$ for $l=3$ and $z_{0}, z_{1}, z_{2}, z_{3}$ for $l=4$. This allows us to use Maschke's convention that in formulas where the subscripts $i, i+1, i+2$ appear, they represent the integers $1,2,3$ in cyclic permutation.

In the description of the basic derivations associated to $\mathscr{B}_{G}$ for $G=$ $G_{32}$ we need additional polynomials defined below:

$$
C_{6}(0)=C_{6}, \quad C_{9}(0)=C_{9}, \quad C_{12}(0)=C_{12}
$$

and for $i=1,2,3$

$$
\begin{aligned}
C_{6}(i)= & z_{0}^{6}+z_{i+1}^{6}+z_{i+2}^{6}+10\left(z_{\imath+1}^{3} z_{i+2}^{3}-z_{0}^{3} z_{i+1}^{3}+z_{0}^{3} z_{i+2}^{3}\right) \\
C_{9}(i)= & z_{i+1}^{3} z_{\imath+2}^{6}+z_{i+1}^{6} z_{\imath+2}^{3}+z_{0}^{3} z_{i+1}^{6}-z_{0}^{6} z_{\imath+1}^{3}-z_{0}^{3} z_{i+2}^{6}-z_{0}^{6} z_{\imath+2}^{3} \\
C_{12}(i)= & z_{0}^{12}+z_{i+1}^{12}+z_{i+2}^{12}-4\left(z_{i+1}^{3} z_{\imath+2}^{9}+z_{i+1}^{9} z_{i+2}^{3}+z_{0}^{3} z_{i+2}^{9}+z_{0}^{9} z_{i+2}^{3}\right. \\
& \left.-z_{0}^{3} z_{i+1}^{9}-z_{0}^{9} z_{i+1}^{3}\right)+6\left(z_{0}^{6} z_{i+1}^{6}+z_{0}^{6} z_{\imath+2}^{6}+z_{i+1}^{6} z_{i+2}^{6}\right) \\
& -228\left(z_{0}^{6} z_{\imath+1}^{3} z_{i+2}^{3}+z_{0}^{3} z_{i+1}^{6} z_{i+2}^{3}-z_{0}^{3} z_{\imath+1}^{3} z_{i+2}^{6}\right) .
\end{aligned}
$$

For the Coxeter groups $D_{3}, B_{3}, A_{4}$ we found flat basic sets $\mathscr{B}_{W^{*}}=\left\{f_{1}^{W}, \cdots, f_{l}^{W}\right\}$ 
using free parameters as described in Section 4. We checked that these basic sets agree, up to multiplication of each $f_{i}^{W}$ by a constant $c_{i}$, with the flat basic sets found by Saito, Sekiguchi and Yano in [16].

(A.4) The pair $\left(G_{25}, D_{3}\right)$. For $W=D_{3}$ we choose the flat basic set $\mathscr{B}_{W}$ :

$$
\begin{aligned}
& f_{1}^{W}=z_{1}^{2}+z_{2}^{2}+z_{3}^{2}, \\
& f_{2}^{W}=8 z_{1} z_{2} z_{3}, \\
& f_{3}^{W}=6\left(z_{1}^{2} z_{2}^{2}+z_{2}^{2} z_{3}^{2}+z_{3}^{2} z_{1}^{2}\right)-\left(z_{1}^{4}+z_{2}^{4}+z_{3}^{4}\right) .
\end{aligned}
$$

This gives the discriminant matrix

$$
\Delta_{W}\left(T_{1}, T_{2}, T_{3} ; \mathscr{B}_{W}\right)=\left[\begin{array}{ccc}
2 T_{1} & 3 T_{2} & 4 T_{3} \\
3 T_{2} & 4 T_{1}^{2}+4 T_{3} & 10 T_{1} T_{2} \\
4 T_{3} & 10 T_{1} T_{2} & 8 T_{1}^{3}+6 T_{2}^{2}
\end{array}\right] .
$$

For $G=G_{25}$ a basic set $\mathscr{B}_{G} \sim \mathscr{B}_{W}$ is given by:

$$
\begin{aligned}
& f_{1}^{G}=C_{6}, \\
& f_{2}^{G}=32 \sqrt{3} C_{9} . \\
& f_{3}^{G}=5 C_{6}^{2}-8 C_{12} .
\end{aligned}
$$

The basic derivations (2.18) associated to $\mathscr{B}_{G}$ are:

$$
\begin{aligned}
& \theta_{1}=\sum_{i=1}^{3} z_{\imath} D_{i} . \\
& \theta_{2}=8 \sqrt{3} \sum_{i=1}^{3}\left(z_{i+2}^{3}-z_{i+1}^{3}\right) z_{i} D_{i}, \\
& \theta_{3}=6 \sum_{i=1}^{3}\left(-z_{i}^{6}+7\left(z_{i+1}^{6}+z_{i+2}^{6}\right)-14 z_{\imath}^{3}\left(z_{\imath+1}^{3}+z_{\imath+2}^{3}\right)+42 z_{\imath+1}^{3} z_{\imath+2}^{3}\right) z_{i} D_{i} .
\end{aligned}
$$

(A.5) The pair $\left(G_{26}, B_{3}\right)$. For $W=B_{3}$ we choose the flat basic set $\mathscr{B}_{W}:$

$$
\begin{aligned}
& f_{1}^{W}= z_{1}^{2}+z_{2}^{2}+z_{3}^{2}, \\
& f_{2}^{W}=3\left(z_{1}^{4}+z_{2}^{4}+z_{3}^{4}\right)-6\left(z_{1}^{2} z_{2}^{2}+z_{2}^{2} z_{3}^{2}+z_{3}^{2} z_{1}^{2}\right), \\
& f_{3}^{W}=\frac{14}{3}\left(z_{1}^{6}+z_{2}^{6}+z_{3}^{6}\right)-10\left(z_{1}^{4} z_{2}^{2}+z_{2}^{4} z_{3}^{2}+z_{3}^{4} z_{1}^{2}+z_{1}^{2} z_{2}^{4}+z_{2}^{2} z_{3}^{4}+z_{3}^{2} z_{1}^{4}\right) \\
& \quad+100 z_{1}^{2} z_{2}^{2} z_{3}^{2} .
\end{aligned}
$$

This gives the the discriminant matrix 


$$
\boldsymbol{\Delta}_{W}\left(T_{1}, T_{2}, T_{3} ; \mathscr{B}_{W}\right)=\left[\begin{array}{ccc}
2 T_{1} & 4 T_{2} & 6 T_{3} \\
4 T_{2} & 8 T_{1}^{3}+12 T_{1} T_{2}+6 T_{3} & 32 T_{1}^{2} T_{2}+8 T_{2}^{2} \\
6 T_{3} & 32 T_{1}^{2} T_{2}+8 T_{2}^{2} & 32 T_{1}^{5}+40 T_{1} T_{2}^{2}
\end{array}\right]
$$

For $G=G_{26}$ a basic set $\mathscr{B}_{G} \sim \mathscr{B}_{W}$ is given by:

$$
\begin{aligned}
& f_{1}^{G}=C_{6}, \\
& f_{2}^{G}=12 C_{12}-3 C_{6}^{2}, \\
& f_{3}^{G}=96 C_{18}+18 C_{6}^{3}-72 C_{6} C_{12} .
\end{aligned}
$$

The basic derivations (2.18) associated to $\mathscr{B}_{G}$ are:

$$
\begin{aligned}
\theta_{1}= & \sum_{i=1}^{3} z_{i} D_{i} . \\
\theta_{2}= & 18 \sum_{i=1}^{3}\left(z_{i}^{6}-3\left(z_{\imath+1}^{6}+z_{\imath+2}^{6}\right)+2 z_{i}^{3}\left(z_{\imath+1}^{3}+z_{i+2}^{3}\right)-26 z_{i+1}^{3} z_{i+2}^{3}\right) z_{i} D_{i} . \\
\theta_{3}= & 18 \sum_{i=1}^{3}\left(7 z_{i}^{12}+28 z_{i}^{9}\left(z_{\imath+1}^{3}+z_{\imath+2}^{3}\right)+162 z_{i}^{6}\left(z_{\imath+1}^{6}+z_{\imath+2}^{6}\right)\right. \\
& -1236 z_{\imath}^{6} z_{i+1}^{3} z_{\imath+2}^{3}+2580 z_{i}^{3}\left(z_{i+1}^{6} z_{\imath+2}^{3}+z_{i+1}^{3} z_{i+2}^{6}\right)-308 z_{\imath}^{3}\left(z_{i+1}^{9}+z_{\imath+2}^{9}\right) \\
& \left.-17\left(z_{\imath+1}^{12}+z_{i+2}^{12}\right)+676\left(z_{i+1}^{9} z_{\imath+2}^{3}+z_{i+1}^{3} z_{i+2}^{9}\right)+90 z_{i+1}^{6} z_{i+2}^{6}\right) z_{i} D_{i} .
\end{aligned}
$$

(A.6) The pair $\left(G_{32}, A_{4}\right)$. For $W=A_{4}$ use the notation

$$
s_{k}=z_{0}^{k}+z_{1}^{k}+z_{2}^{k}+z_{3}^{k}+(-1)^{k}\left(z_{0}+z_{1}+z_{2}+z_{3}\right)^{k} .
$$

We choose the fiat basic set $\mathscr{B}_{W}$ :

$$
\begin{aligned}
& f_{1}^{W}=\frac{1}{2} s_{2}, \\
& f_{2}^{W}=\frac{\sqrt{10}}{3} s_{3}, \\
& f_{3}^{W}=\frac{5}{2} s_{4}-\frac{3}{4} s_{2}^{2} . \\
& f_{4}^{W}=2 \sqrt{10}\left(s_{5}-\frac{2}{3} s_{3} s_{3}\right) .
\end{aligned}
$$

This gives the discriminant matrix

$$
\begin{aligned}
\Delta_{W}\left(T_{1}, T_{2}, T_{3}, T_{4} ; \mathscr{B}_{W}\right) & \\
\quad= & \left(\begin{array}{cccc}
2 T_{1} & 3 T_{2} & 4 T_{3} & 5 T_{4} \\
3 T_{2} & 4 T_{3}+4 T_{1}^{2} & 5 T_{4}+10 T_{1} T_{2} & 12 T_{1} T_{3}+6 T_{2}^{2} \\
4 T_{3} & 5 T_{4}+10 T_{1} T_{2} & 12 T_{1} T_{3}+12 T_{2}^{2}+8 T_{1}^{3} & 14 T_{2} T_{3}+28 T_{1}^{2} T_{2} \\
5 T_{4} & 12 T_{1} T_{3}+6 T_{2}^{2} & 14 T_{2} T_{3}+28 T_{1}^{2} T_{2} & 8 T_{3}^{2}+32 T_{1} T_{2}^{2}+16 T_{1}^{4}
\end{array}\right)
\end{aligned}
$$


For $G=G_{32}$ a basic set $\mathscr{B}_{G} \sim \mathscr{B}_{W}$ is given by

$$
\begin{aligned}
f_{1}^{G} & =F_{12}, \\
f_{2}^{G} & =\frac{4}{3} F_{18}, \\
f_{3}^{G} & =21 F_{12}^{2}-25 F_{24}, \\
f_{4}^{G} & =\frac{8}{5}\left(11 F_{12} F_{18}-25 F_{30}\right) .
\end{aligned}
$$

The basic derivations (2.18) associated to $\mathscr{B}_{G}$ are:

$$
\begin{aligned}
\theta_{1}= & \sum_{i=0}^{3} z_{i} D_{i} \\
\theta_{2}= & 6 \sum_{i=0}^{3}\left(-3 z_{i}^{6}+7 C_{6}(i)\right) z_{i} D_{i} \\
\theta_{3}= & 36 \sum_{i=0}^{3}\left(7 z_{i}^{12}-26 z_{i}^{6} C_{6}(i)+2080 z_{i}^{3} C_{9}(i)+\frac{26}{3} C_{6}(i)^{2}-\frac{65}{3} C_{12}(i)\right) z_{i} D_{i} \\
\theta_{4}= & 216 \sum_{i=0}^{3}\left(-11 z_{i}^{18}+57 z_{i}^{12} C_{6}(i)-3040 z_{i}^{9} C_{9}(i)+722 z_{i}^{6} C_{6}(i)^{2}\right. \\
& -1235 z_{i}^{6} C_{12}(i)+4560 z_{i}^{3} C_{6}(i) C_{9}(i)-\frac{38}{3} C_{6}(i)^{3} \\
& \left.+\frac{95}{3} C_{6}(i) C_{12}(i)+7600 C_{9}(i)^{2}\right) z_{i} D_{i} .
\end{aligned}
$$

\section{REFERENCES}

[1] Etsuko Bannai, Fundamental groups of the spaces of regular orbits of the finite unitary reflection groups of dimension 2, J. Math. Soc. Japan, 28 (1976), 447-454.

[2] H. S. M. Coxeter, The symmetry groups of the regular complex polygons, Arch., Math., 13 (1962), 86-97.

[3] - Finite groups generated by unitary reflections, Abhandlungen Math. Sem. Univ. Hamburg, 31 (1967), 127-135.

[4] — Regular Complex Polytopes, Cambridge Univ. Press, 1974.

[5] P. Deligne, Les immeubles des groupes de tresses généralisés, Invent. math., 17 (1972), 273-302.

[6 ] J. H. Grace and A. Young, The Algebra of Invariants, Cambridge Univ. Press, 1903.

[ 7 ] F. Klein, Vorlesungen Über das Ikosaeder, Teubner, Leipzig, 1884.

[ 8 ] D. W. Koster, Complex Reflection Groups, Thesis, Univ. of Wisconsin, Madison, 1975.

[ 9 ] H. Maschke, Aufstellung des vollen Formensystems einer quaternären Gruppe von 51840 linearen substitutionen, Math. Ann., 33 (1888), 317-344.

[10] J. Milnor, Singular points of complex hypersurfaces, Ann. of Math. Studies 61, Princeton Univ. Press, 1968. 
[11] T. Nakamura, A Note on the $K(\pi, 1)$ property of the orbit space of the unitary reflection group $G(m, l, n)$, Sci. Papers College of Arts and Sciences, Univ. Tokyo, 33 (1983), 1-6.

[12] P. Orlik and L. Solomon, Unitary reflection groups and cohomology, Invent. math., 59 (1980), 77-94.

[13] - Complexes for reflection groups, in Proc. Midwest Algebraic Geometry Conf., Lecture Notes in Math., No. 862, Springer Verlag (1981), 193-207.

[14] - The Hessian map in the invariant theory of reflection groups, Nagoya Math. J., 109 (1988), 1-21.

[15] K. Saito, On a Linear Structure of a Quotient Variety by a Finite Reflexion Group, RIMS Report 288, Kyoto 1979.

[16] K. Saito, T. Yano and J. Sekiguchi, On a certain generator system of the ring of invariants of a finite reflection group, Comm. Algebra, 8 (1980), 373-408.

[17] G. C. Shephard, Regular complex polytopes, Proc. London Math. Soc., (3) 2 (1952), 82-97.

[18] - Unitary groups generated by reflections, Canad. J. Math., 5 (1953), 364-383.

[19] G. C. Shephard and J. A. Todd, Finite unitary reflection groups, Canad. J. Math., 6 (1954), 274-304.

[20] T. A. Springer, Regular elements of finite reflection groups, Invent. math., 25 (1974), 159-198.

[21] - Invariant Theory, Lecture Notes in Math., No. 585, Springer Verlag, 1977.

[22] H. Terao, Discriminant of a holomorphic map and logarithmic vector fields, J. Fac. Sci. Univ. Tokyo, Sect. IA Math., 30 (1983), 379-391.

[23] H. W. Turnbull, The Theory of Determinants, Matrices and Invariants, 3rd Ed., Dover 1960.

[24] T. Yano and J. Sekiguchi, The microlocal structure of weighted homogeneous polynomials associated with Coxeter systems I, II, Tokyo J. Math., 2 (1979), $193-219 ; 4$ (1981), 1-34.

University of Wisconsin

Madison, WI 53706, U.S.A. 